Supporting information

\title{
Continuous Processing of Cellulose Nanofibril Sheets Through Conventional Single-Screw
} Extrusion

Authors: Sami M. El Awad Azrak ${ }^{\mathrm{a}}$, William J. Costakis ${ }^{\mathrm{a}}$, Robert J. Moon ${ }^{\mathrm{b}}$, Gregory T.

Schueneman ${ }^{\mathrm{b}}$, and Jeffrey P. Youngblood, ${ }^{\mathrm{a}}{ }^{*}$

Authors addresses:

a. School of Materials Engineering, Purdue University, West Lafayette, IN 47907, United States of America

b. Forest Products Laboratory, United States Forest Service, Madison, Wisconsin 53726, United States of America

*Corresponding authors

Email: jpyoungb@purdue.edu, Phone: +1 765-496-2294, Fax: +1 765 494-1204 

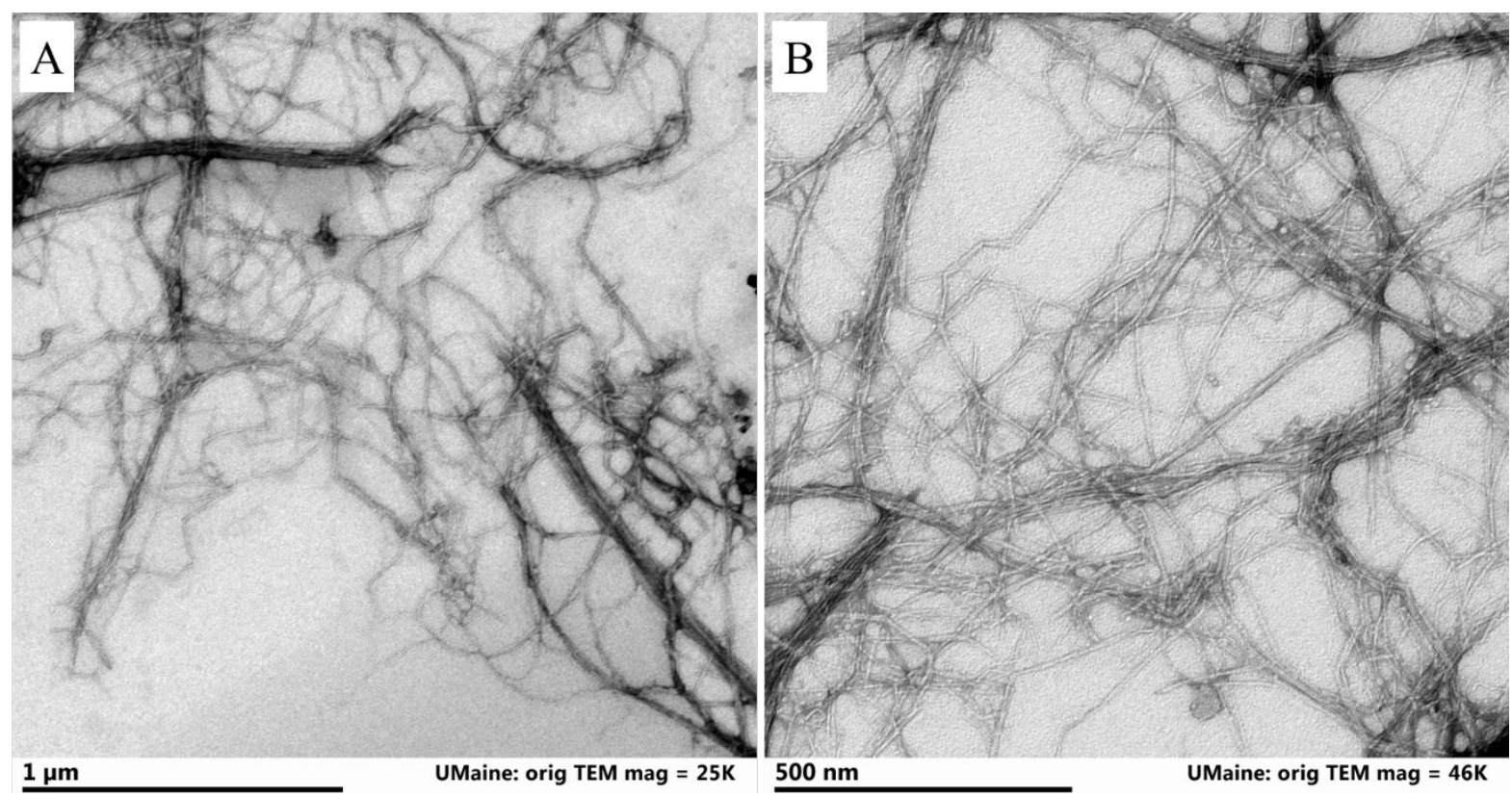

Figure S1. TEM images at two different magnifications $(A=25,000 x$, and $B=46,000 x)$ of the mechanically fibrillated CNFs produced at the Process Development Center (PDC), University of Maine, Orono, ME, USA. The CNF material was produced by fibrillating northern bleached softwood Kraft pulp via a multistage disk refiner. The TEM images were taken from a previous batch of CNF, yet both batches of CNF were produced the same way.

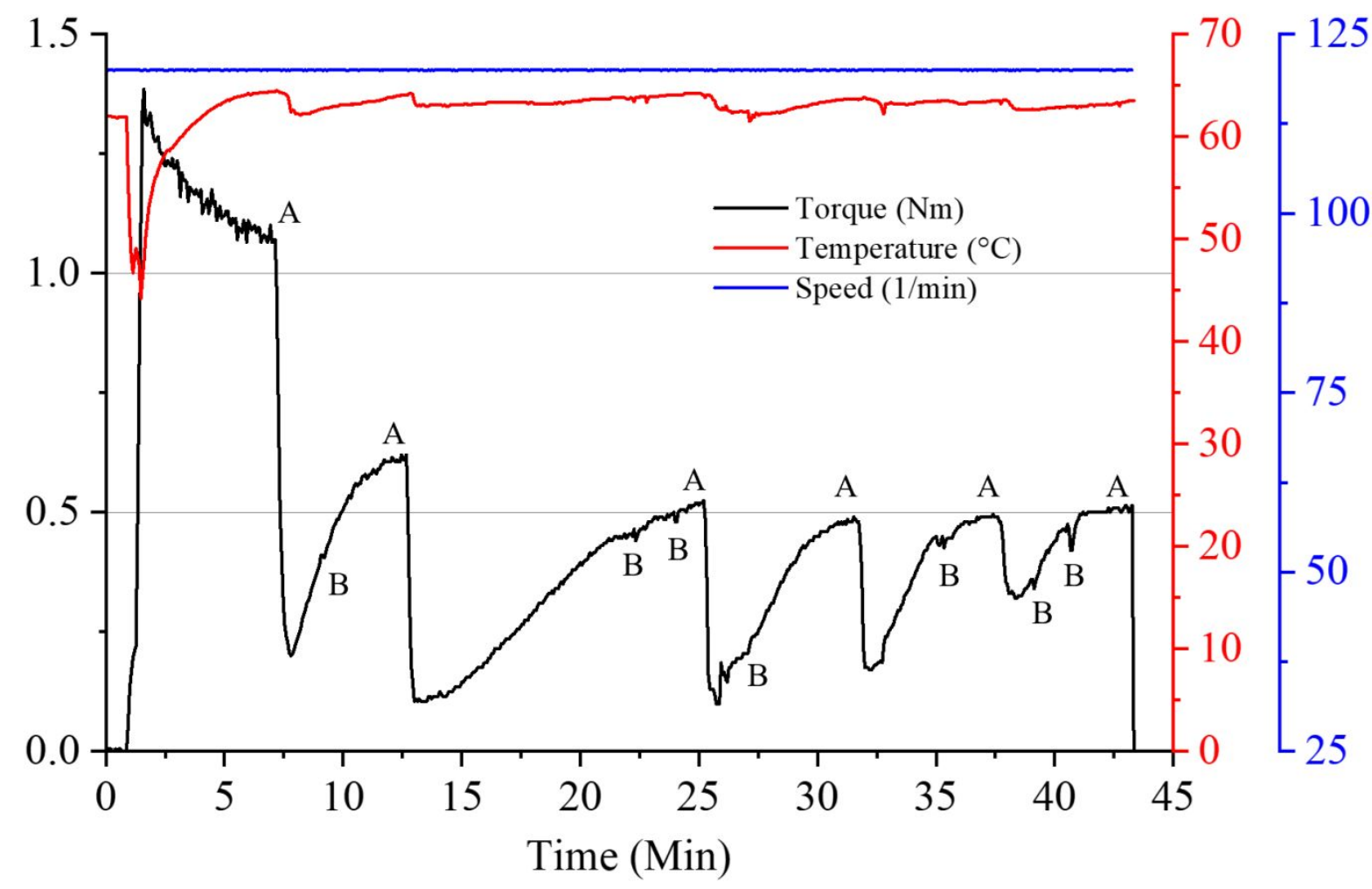

Figure S2. Recorded torque $(\mathrm{Nm})$, temperature $\left({ }^{\circ} \mathrm{C}\right)$, and mixing speed $(\mathrm{rpm})$ during mixing of a $\mathrm{CNF} / \mathrm{CMC}$ paste in a Brabender high shear torque mixer. The "A" points mark the addition of CMC while the "B" points mark the addition of water. The final paste solids concentration was $15.32 \mathrm{wt} \%$ with a CMC to CNF dry weight ratio of $0.1: 1$. The plateau represents complete polymer incorporation into the paste and depends on the added polymer and final solids concentration of the paste. 


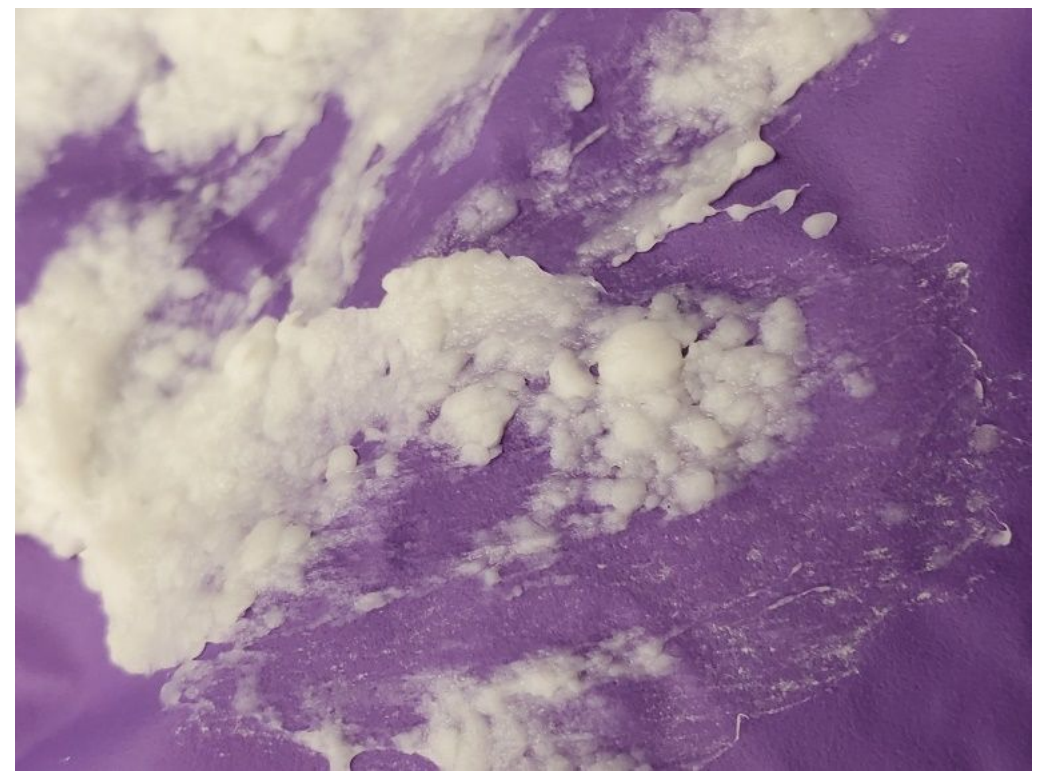

Figure S3.CNF/aPAM paste mixed in the Brabender at a dry weight ratio of 0.05:1. Clumps or grainy texture can be clearly appreciated instead of a homogenous/creamy texture. The torque plateaus for this paste were not reached even though the mixing times was extended for over an hour instead of the typical $\sim 40$ minutes.

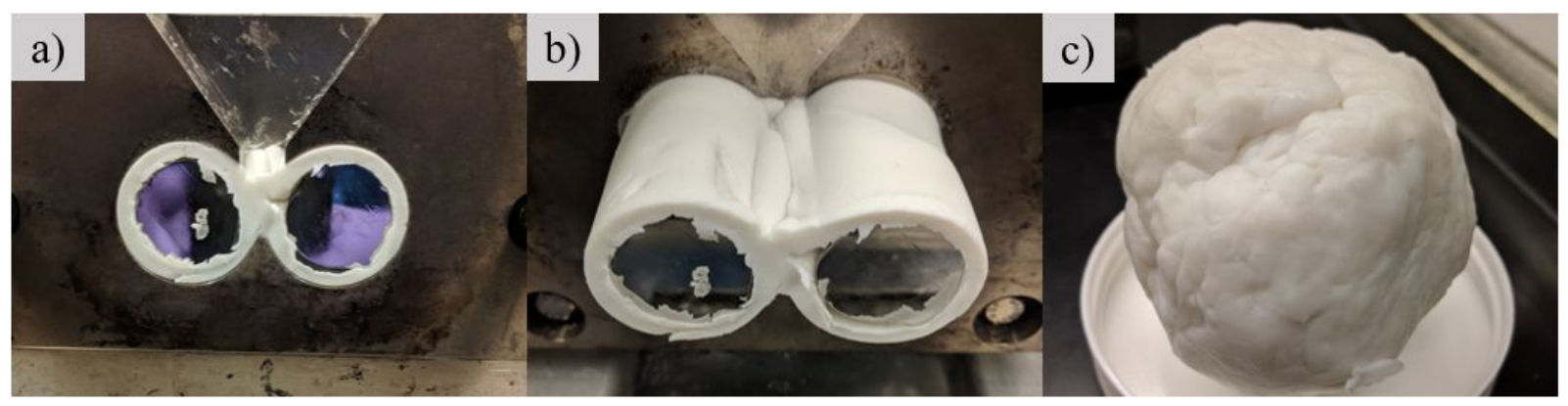

Figure S4. CNF/CMC paste after mixing and complete CMC incorporation inside the mixing chamber (a), wrapped around the mixing Banbury mixing blades (b), and removed from the mixer and shaped into a ball (c). The texture of the paste was very smooth and without any clumps or aggregates.

Table S1. Summary table showing all the processed highly loaded extrusion pastes prepared. These pastes were extruded and hot pressed for mechanical testing. Enough material for each batch was prepared to perform extrusion and rheological analysis.

\begin{tabular}{|c|c|c|}
\hline Extrusion Paste & Mixture Ratio (processing aid to CNF) & $\begin{array}{c}\text { Final Solids Concentration } \\
\text { [wt\%] }\end{array}$ \\
\hline $\mathrm{CNF} / \mathrm{CMC}$ & $0.1: 1$ & 15.32 \\
\hline $\mathrm{CNF} / \mathrm{CMC}$ & $0.1: 1$ & 19.14 \\
\hline $\mathrm{CNF} / \mathrm{CMC}$ & $0.1: 1$ & 25.31 \\
\hline $\mathrm{CNF} / \mathrm{CMC}$ & $0.05: 1$ & 15.32 \\
\hline $\mathrm{CNF} / \mathrm{XG}$ & $0.1: 1$ & 15.32 \\
\hline $\mathrm{CNF} / \mathrm{XG}$ & $0.05: 1$ & 15.32 \\
\hline $\mathrm{CNF} / \mathrm{aPAM}$ & $0.026: 1$ & 15.32 \\
\hline $\mathrm{CNF} / \mathrm{aPAM}$ & $0.013: 1$ & 15.32 \\
\hline
\end{tabular}




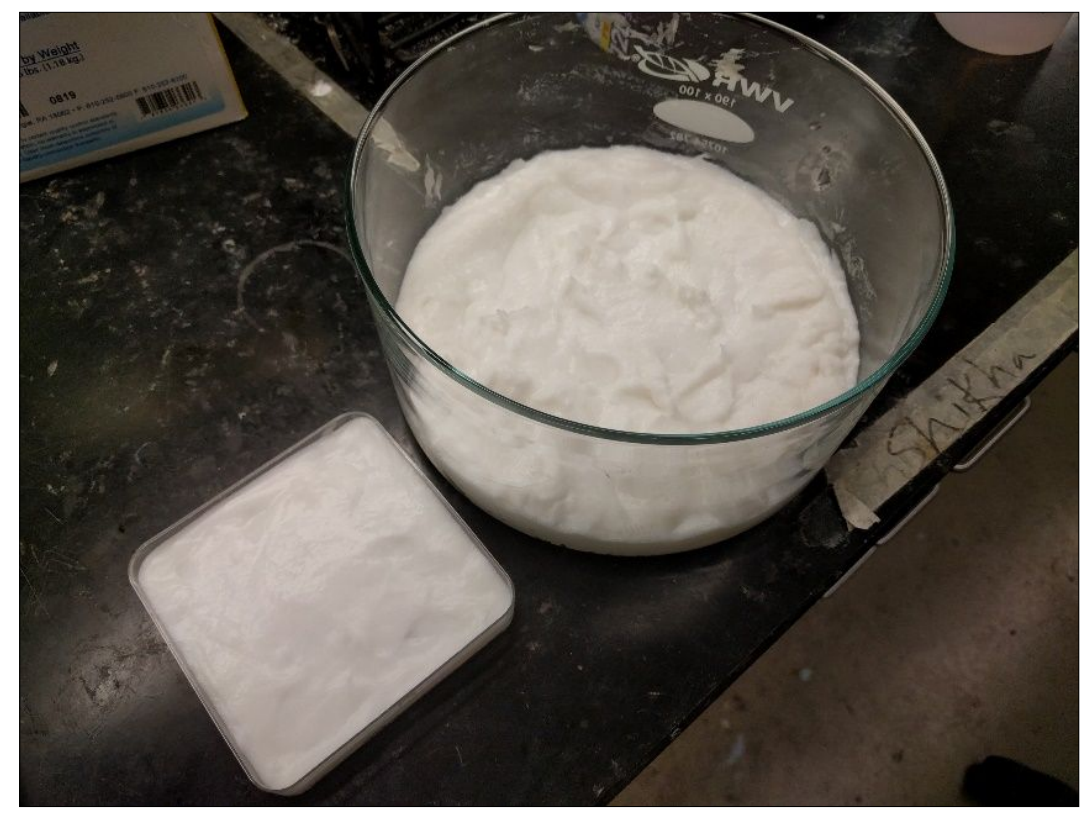

Figure S5.Air dried CNF/CMC paste prepared by air drying the watery CNF+CMC suspension in a humidity controlled oven long periods of time. The suspension was cast in either square polypropylene petri dishes or in a large crystallization dish as shown. The current state of the paste shown is of a $\sim 10 \mathrm{wt} \%$ solids concentration.

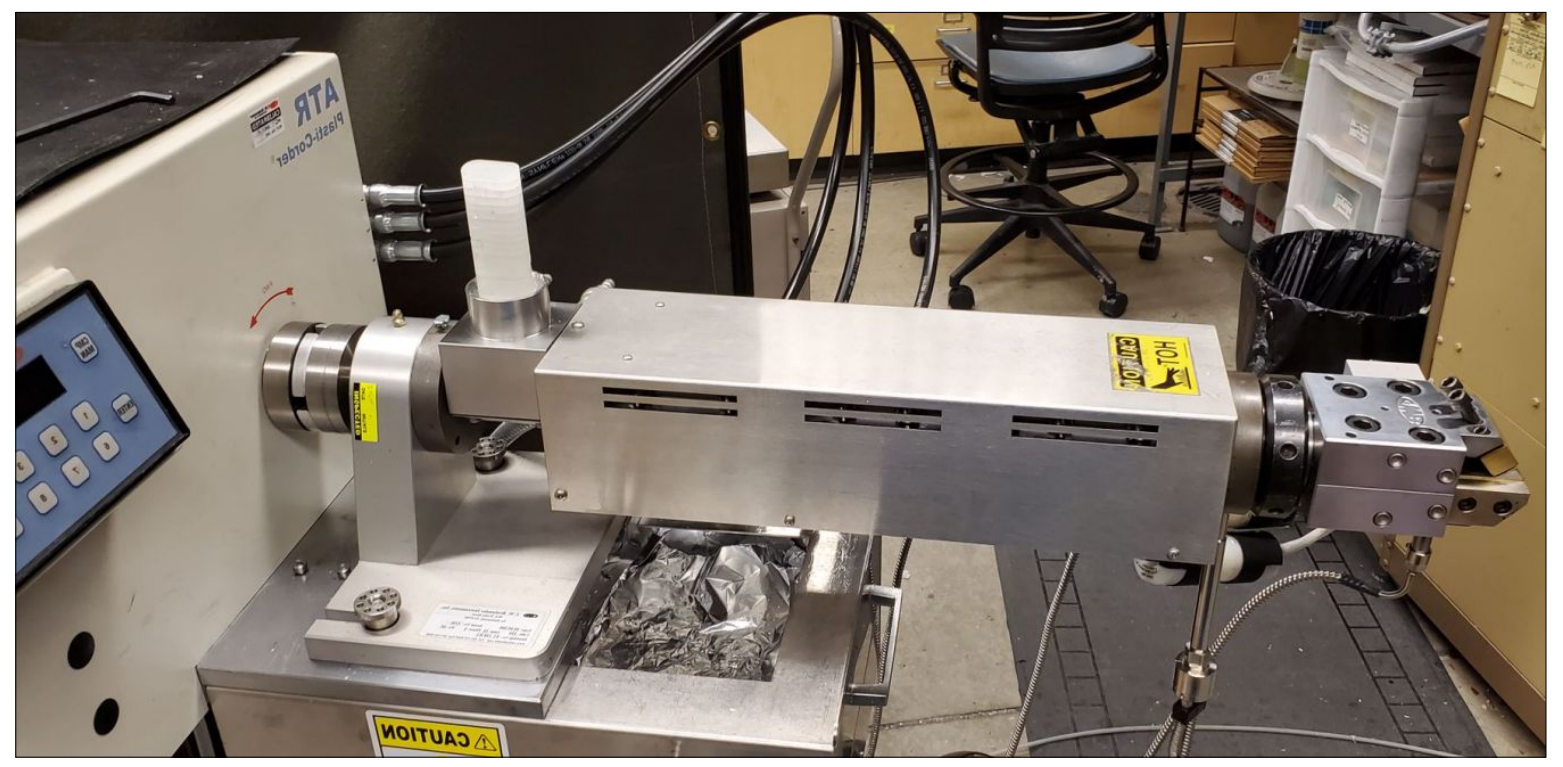

Figure S6. Brabender torque rheometer (ATR) unit but with an attached single screw extruder unit $(\mathrm{L} / \mathrm{D}=25$, barrel diameter of $1.9 \mathrm{~cm}$ (3/4 inch), and 4 independent heating zones). The in-house made plunger, located in the feed section, was used to push the highly viscous $\mathrm{CNF} /$ processing aid pastes. 


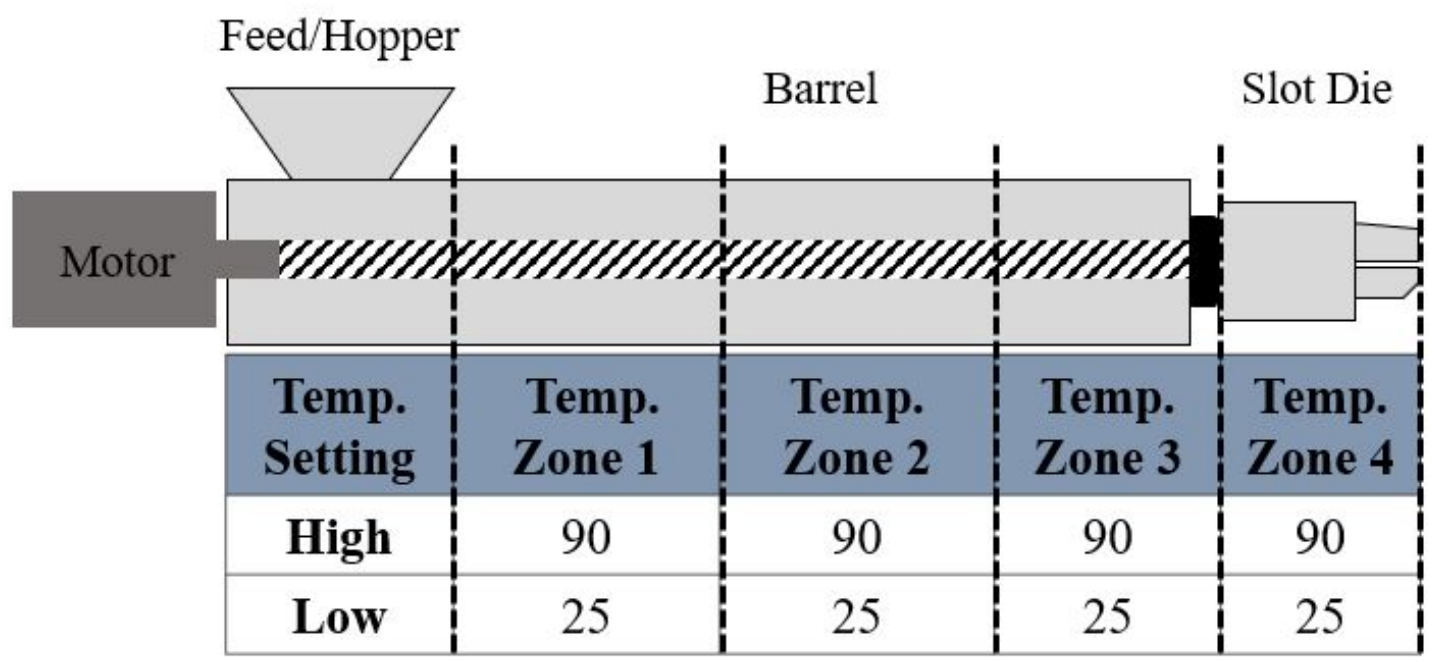

Figure S7. Extruder configuration and processing parameters used to process highly loaded CNF/processing aid and pure CNF into wet sheets. The shown temperature is in ${ }^{\circ} \mathrm{C}$. Unless otherwise stated the screw speed used was $7 \mathrm{rpm}$ for all experiments.

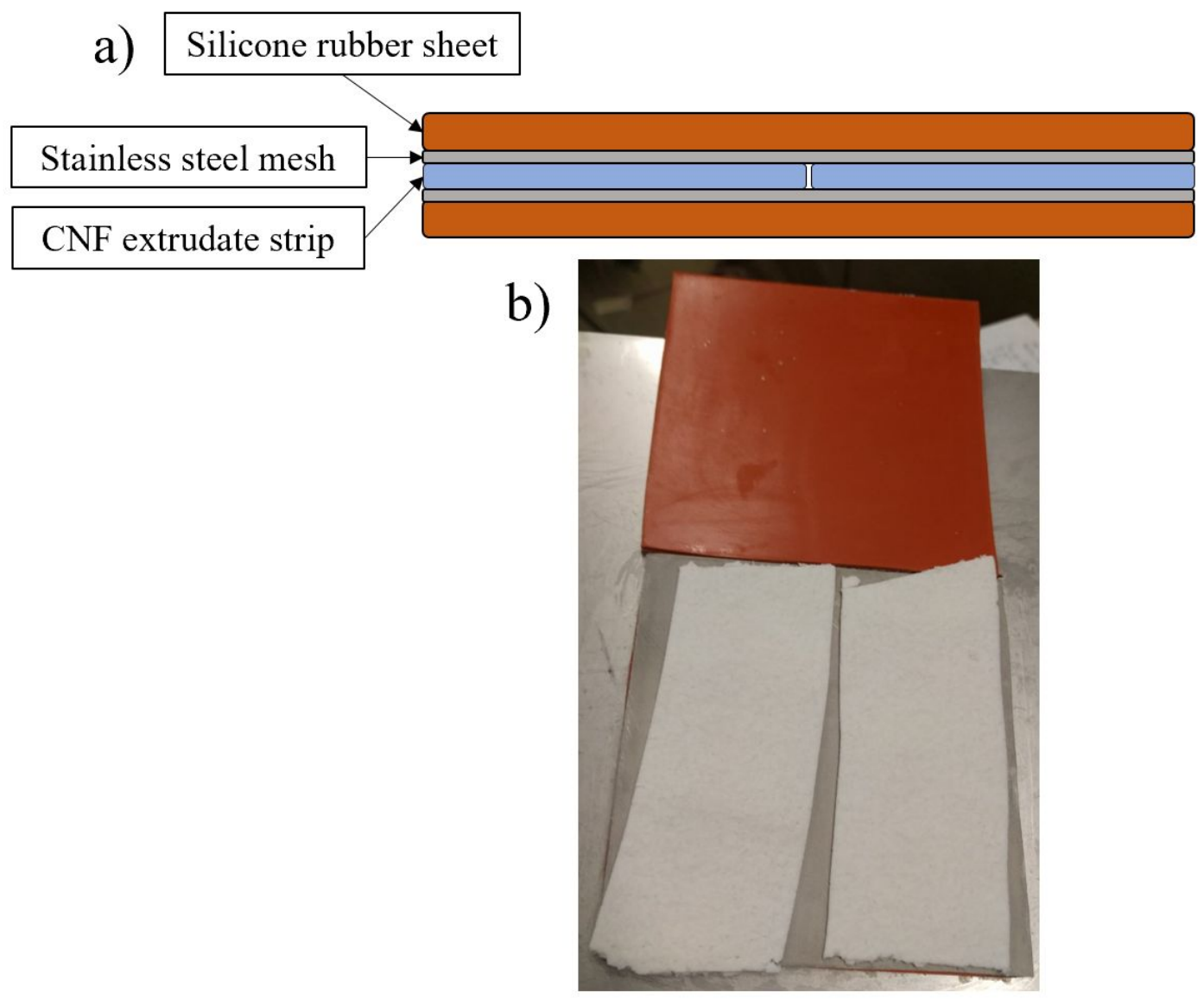

Figure S8. Graphical representation of the pressing configuration used to fully consolidate the extrudates (a) which shows the different components used and b) an actual photograph of a CNF/CMC extrudate before pressing at $126^{\circ} \mathrm{C}$ for 30 minutes. 


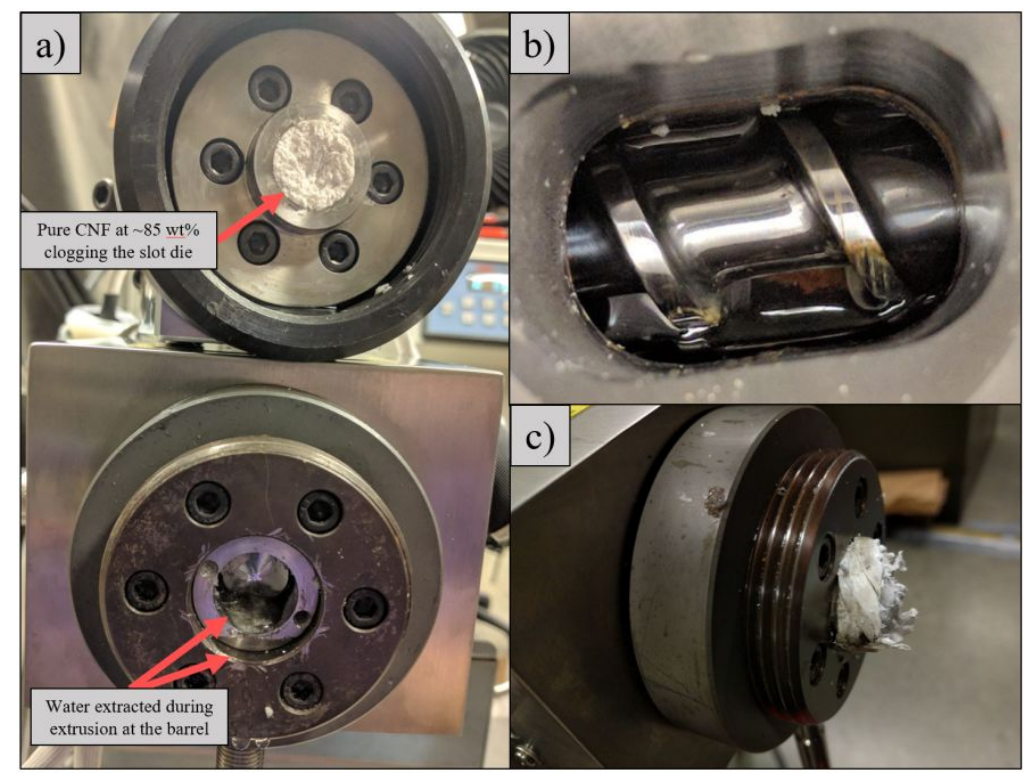

Figure S9. Failed attempt at extrusion of pure CNF with a solids concentration of $17 \mathrm{wt} \%$. The material clogged the entrance of the sheet die (a) and the end of the barrel (c). The clogged and extracted water pooled in the hopped region (b).

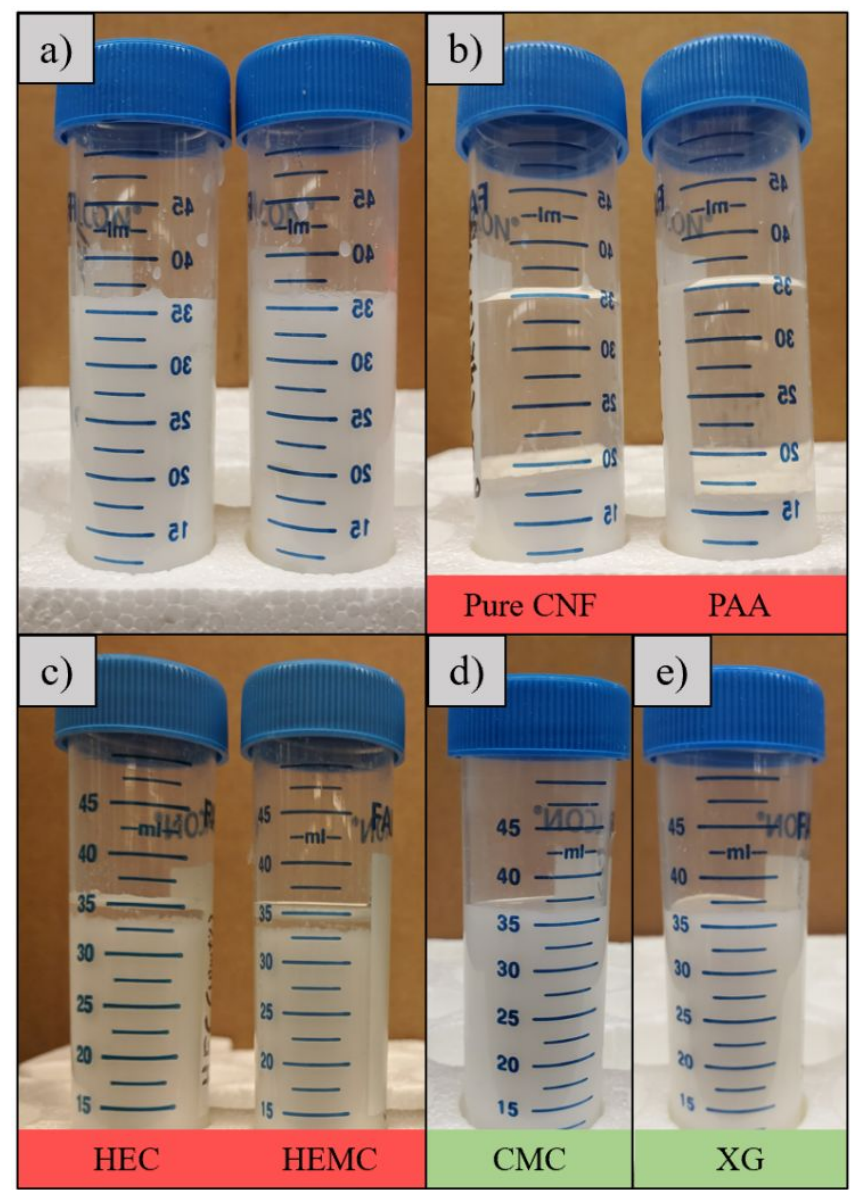

Figure S10. Few examples of centrifuge tubes filled with CNF plus processing aid suspensions or gels (a) before centrifugation and (b, c, d, e) after centrifugation. For pure CNF and PAA roughly $20 \mathrm{ml}$ of water was separated while for HEC and HEMC roughly 2 to $3 \mathrm{ml}$ of water was separated. $\mathrm{CMC}$ and $\mathrm{XG}$ mixtures did not dewater in any perceivable manner. 


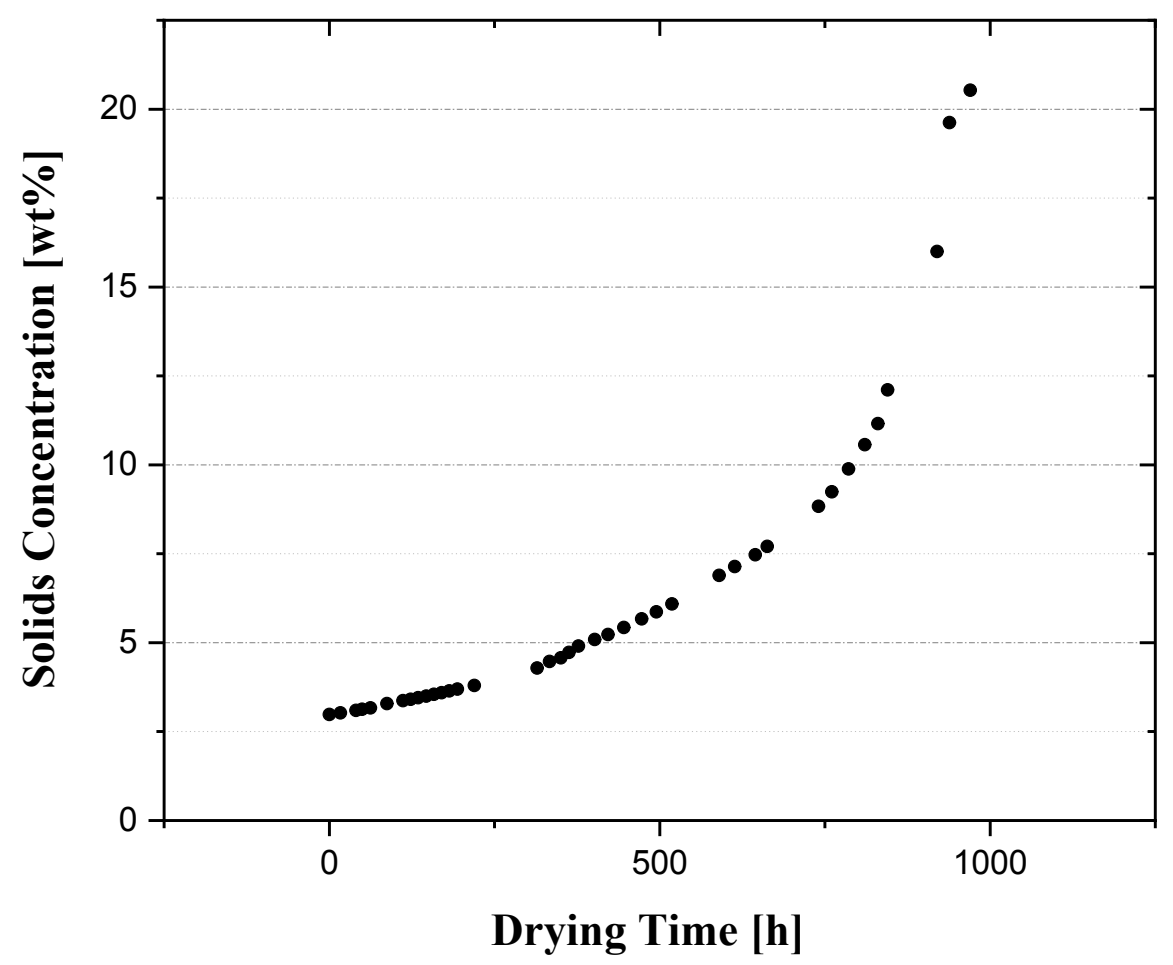

Figure S11. Solids concentration [wt.\%] verses drying time [h] for an air dried CNF/CMC paste with a solids concentration of $\sim 3$ wt. $\%$ in a humidity controlled oven. To achieve a solids concentration of $\sim 15 \mathrm{wt} . \%$ the paste had to be dried for $\sim 40$ days.

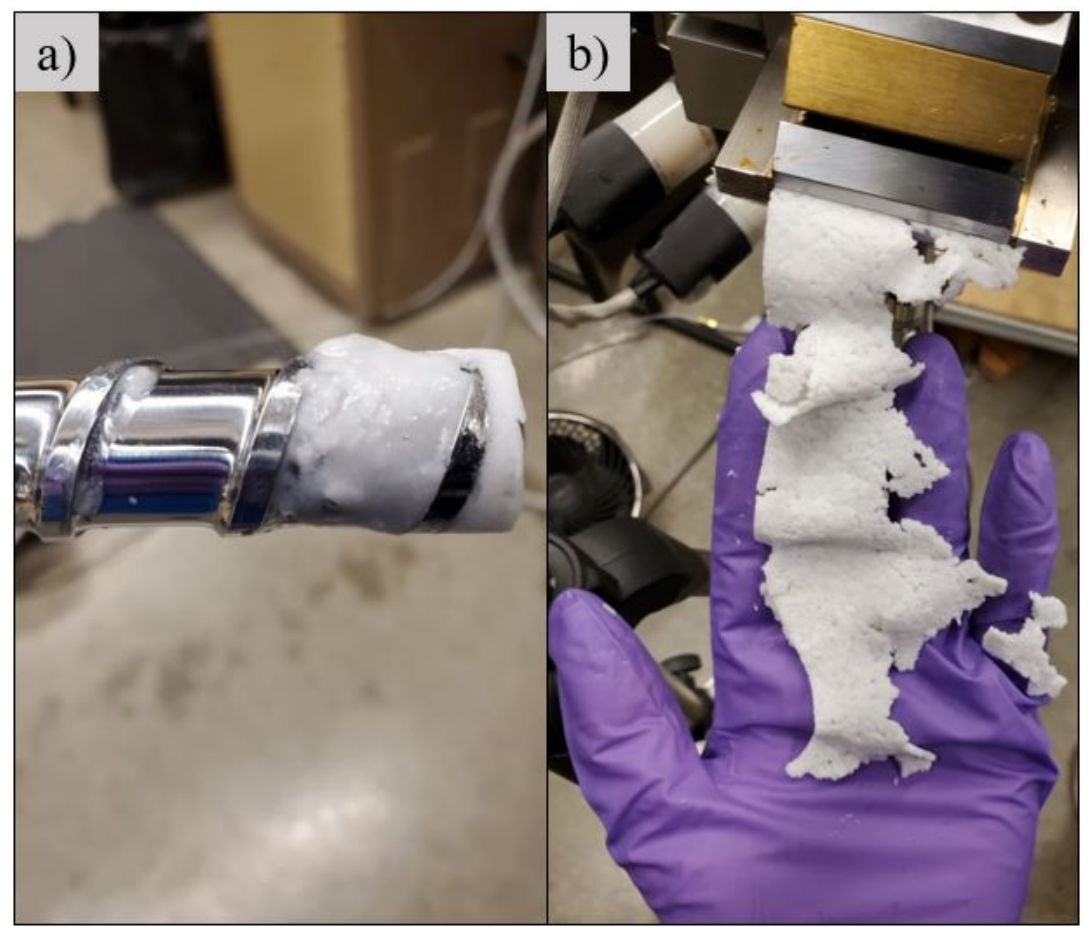

Figure S12. Dewatering observed at the end of the screw after extrusion of a CNF/aPAM paste with an aid to CNF dry weight ratio of 0.013:1 (a), and fractured extrudate during extrusion of the same paste (b). A similar dewatering behavior and formation of a fractured extrudate was experienced for pastes of $\mathrm{CNF} / \mathrm{CMC}$ and $\mathrm{CNF} / \mathrm{XG}$ with a polymer to dry weight ratio of 0.05:1. 


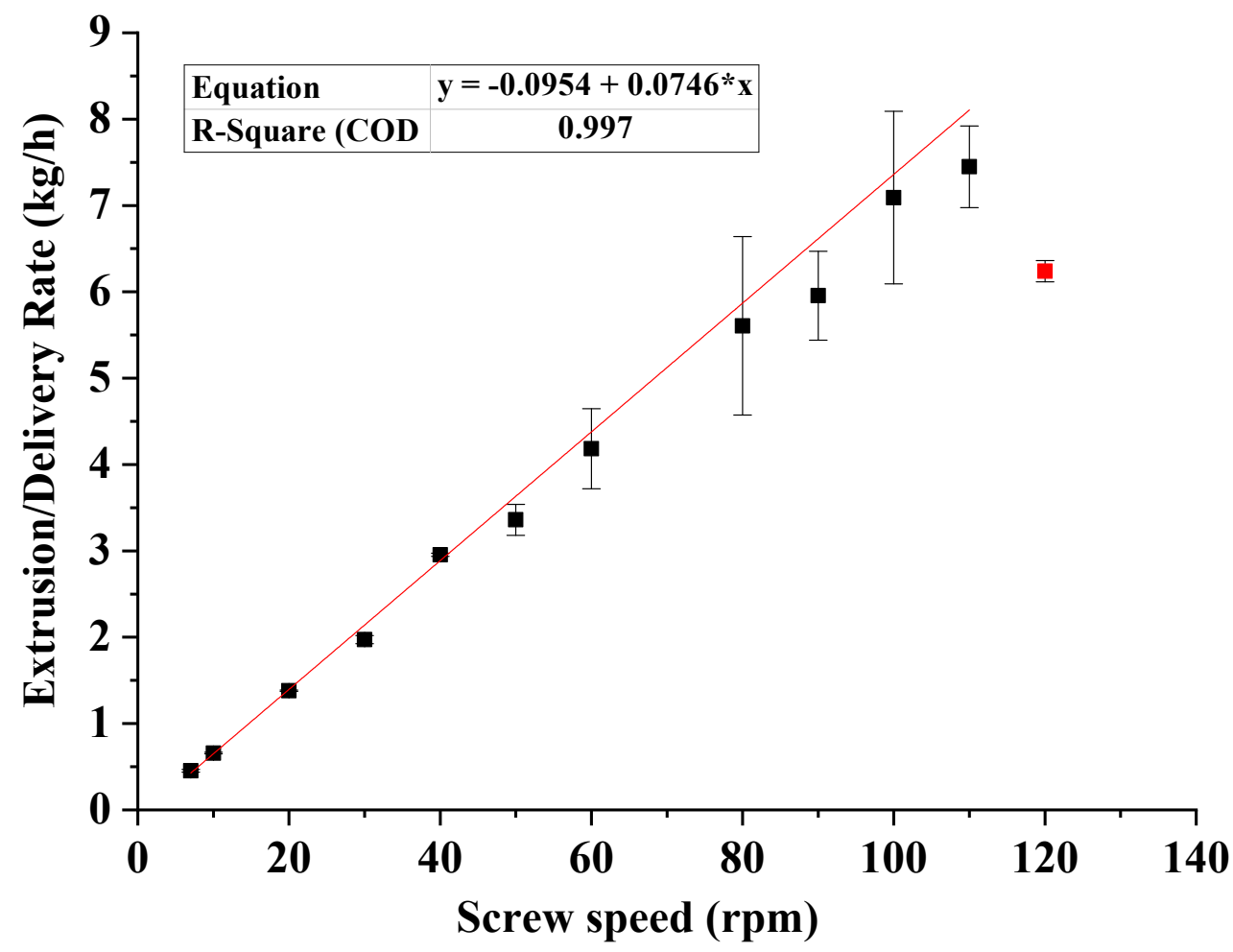

Figure S13. Extrusion delivery rate $[\mathrm{kg} / \mathrm{h}]$ verses screw speed $[\mathrm{rpm}]$ for a CNF/CMC paste with a dry weight ratio of $0.1: 1$ and a solids concentration of $\sim 15 \mathrm{wt} . \%$. Three aliquots of material were collected for each data point. The weight of the sample and delivery time were recorded in real time. A linear fit was applied to the data showing a strong correlation between the two variables $\left(R^{2}=0.997\right)$. The red dot was excluded from the analysis for reasons explained in the manuscript. The error bars represent 1 standard deviation away from the mean.

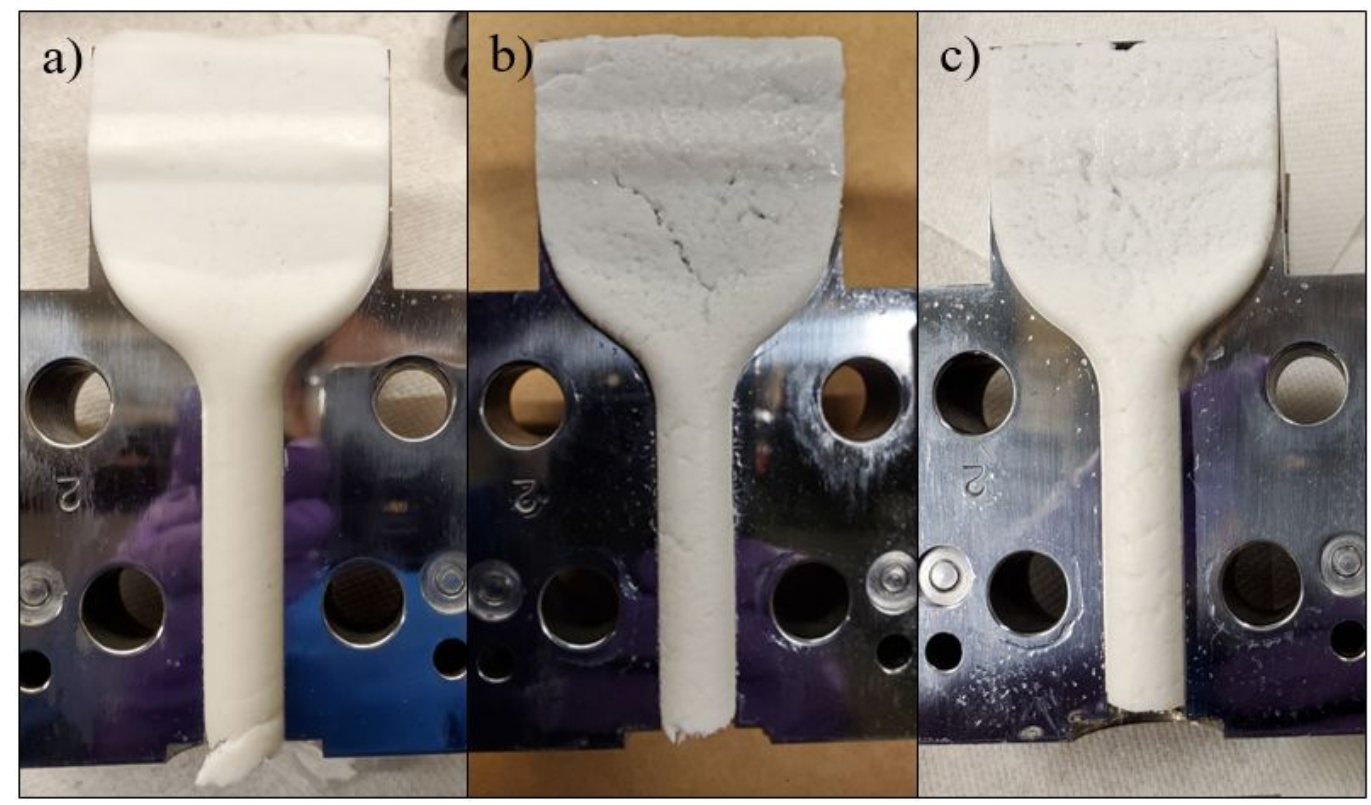

Figure S14. View from the inside of the slot or sheet die after the extrusion of a CNF/CMC paste (a), CNF/XG paste (b), and $\mathrm{CNF} / \mathrm{aPAM}$ paste (c) at a ratio of 0.1:1 for $\mathrm{CNF} / \mathrm{CMC}$ and $\mathrm{CNF} / \mathrm{XG}$ and a ratio of $0.026: 1$ for CNF/aPAM. 


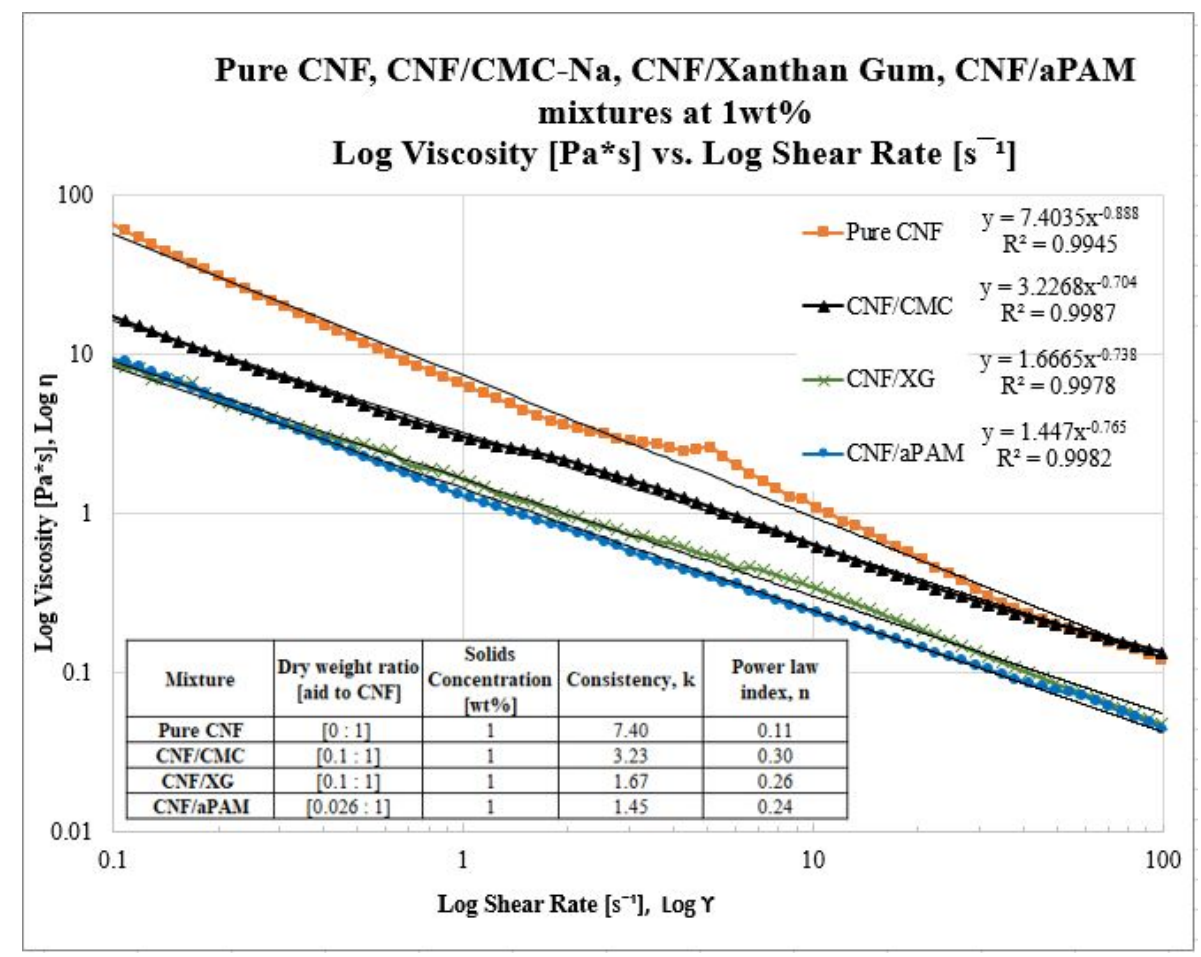

Figure S15. Log of apparent viscosity $\left[\mathrm{Pa}^{*} \mathrm{~s}\right]$ verses $\log$ of shear rate $\left[\mathrm{s}^{-1}\right]$ for suspensions of pure $\mathrm{CNF}, \mathrm{CNF} / \mathrm{CMC}, \mathrm{CNF} / \mathrm{XG}$, and CNF/aPAM. The table inset shows important rheological parameters computed from the power law fit on the obtained data. The equation on the top right corner represent the power law models.

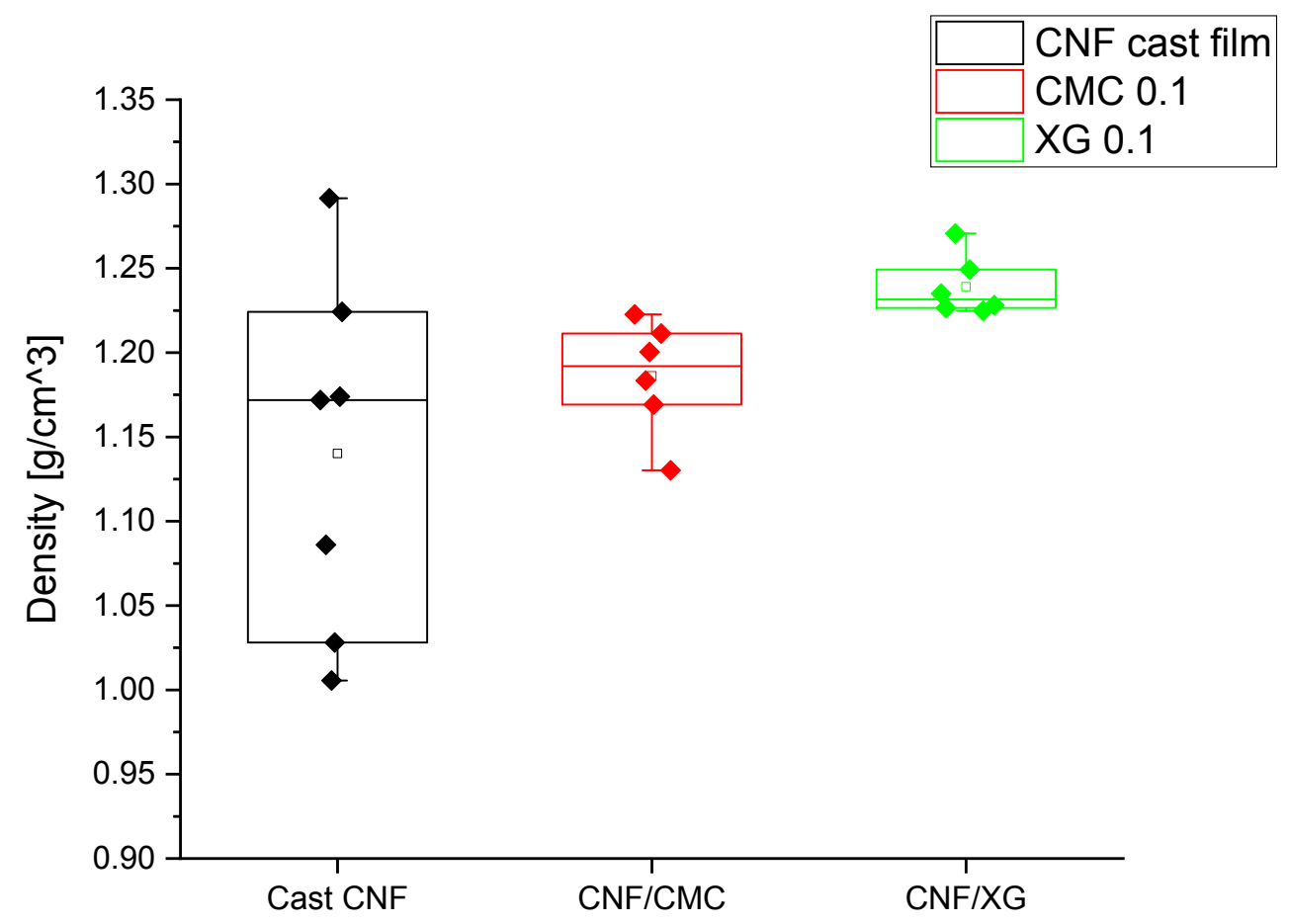

Figure S16. Boxplot of the density data for cast CNF films and extruded sheets of CNF/CMC and CNF/XG. The filled points represent the actual sample units while the middle unfilled square represents the mean of the sample. 


\section{Thermogravimetric Analysis of pressed extrudates}

Thermogravimetric analysis (TGA) of the pressed and heated extrudates revealed a similar degradation behavior between $\mathrm{CNF} / \mathrm{aPAM}$ and the $\sim 30 \mathrm{wt} . \%$ pure $\mathrm{CNF}$ extrudate with a pronounced weight loss reaching over $2 \%$ at $100^{\circ} \mathrm{C}$ (Figure S17). On the other hand, $\mathrm{CNF} / \mathrm{CMC}$ and $\mathrm{CNF} / \mathrm{XG}$ sheets behaved more like cast $\mathrm{CNF}$ films with a less pronounced weight loss reaching $1 \%$ at $100^{\circ} \mathrm{C}$. For all cases, the weight loss could be linked to how well the material retains water as there is always some amount of free and bound water left and CNF starts degrading at $180^{\circ} \mathrm{C}$ while aids like $\mathrm{CMC}$ start degrading at $200^{\circ} \mathrm{C}^{1-3}$. $\mathrm{CMC}$ and $\mathrm{XG}$ could have acted as a stronger CNF dispersant (i.e., reduced agglomerates) and thus enabled more surface area making it harder for water to escape through a more homogenous structure. This is in contrast to CNF/aPAM and pure extruded CNF sheets whose structure looked heavily agglomerated even in the macroscopic state (Figure 1 and Figure $4 \mathrm{f}$ in the manuscript). For example, TEMPO oxidized CNF with a higher fibrillar dispersion and smaller fibril diameters form much stronger and more viscous gels which binds or retains water more strongly ${ }^{4,5}$. Furthermore, cast CNF films achieve a good level of homogeneity as the drying process is very slow compared to pressing which allows for CNF fibrils to find the best possible packing before gelation occurs and movement is restricted.

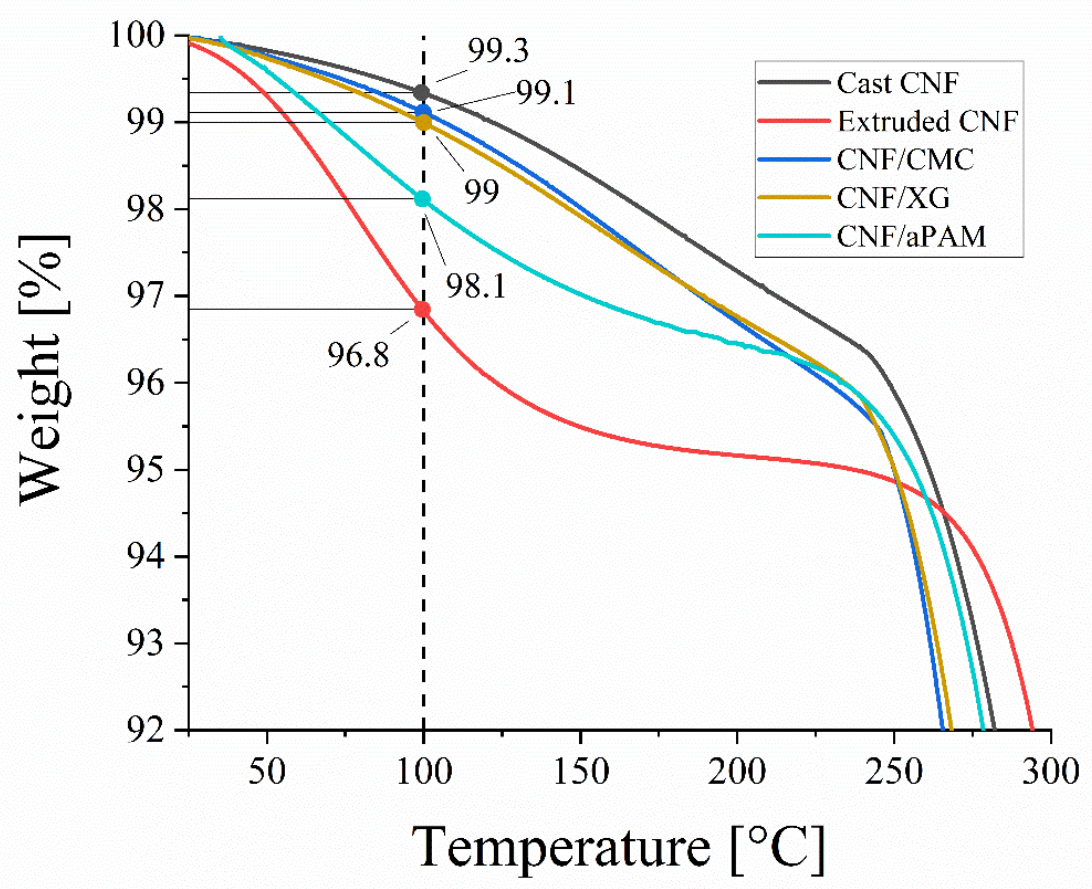

Figure S17. Thermogravimetric analysis of cast CNF, extruded pure CNF, CNF/CMC, CNF/XG, and CNF/aPAM. The boiling point of water $\left(100^{\circ} \mathrm{C}\right)$ was selected as a comparison point. Prior to testing all the samples were preconditioned for over a week at $25 \%$ RH. 


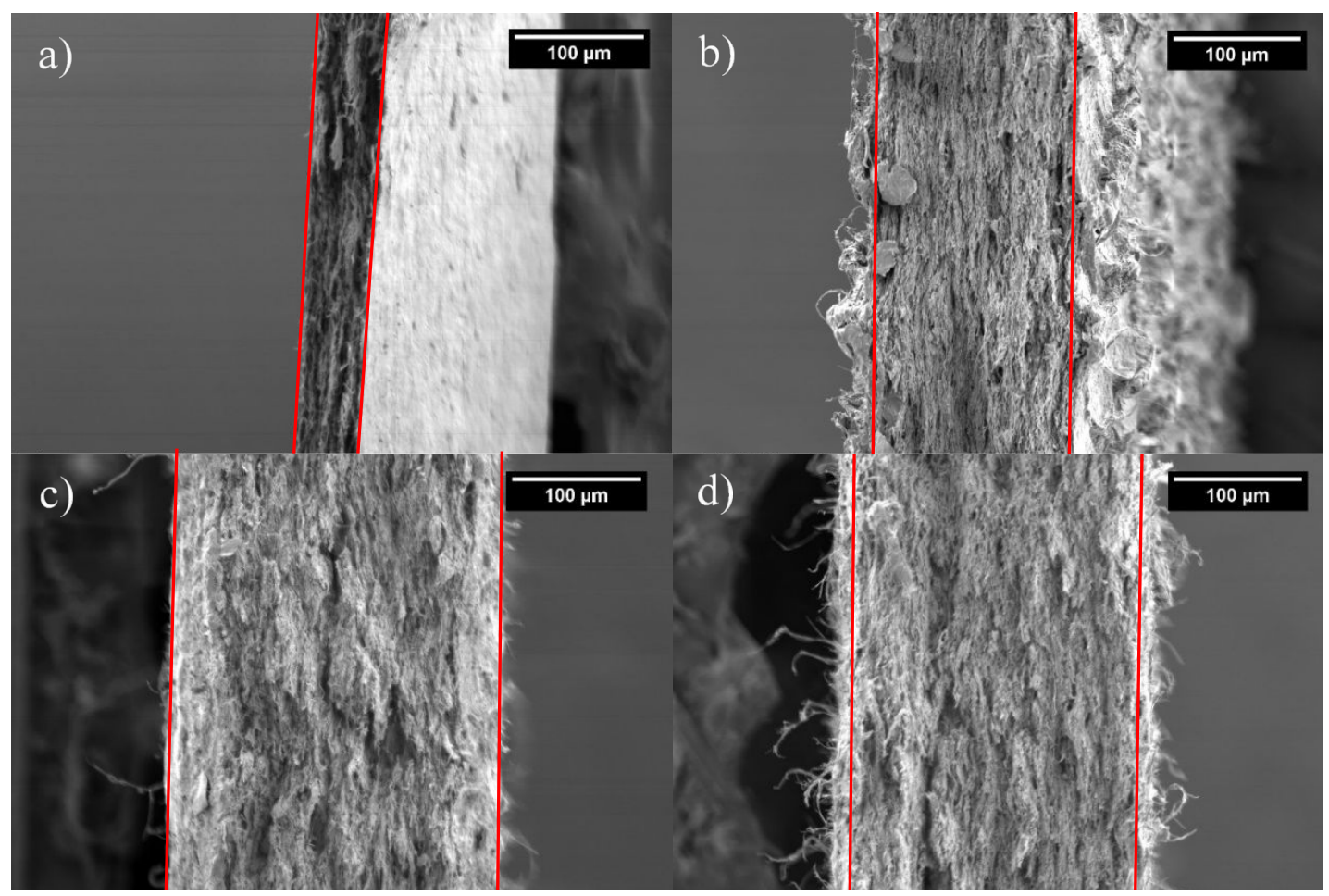

Figure S18. Scanning electron microscopy of the fractured surfaces of cast CNF (a), CNF/CMC at 0.1:1 (b), CNF/XG at 0.1:1 (c), and $\mathrm{CNF} / \mathrm{XG}(\mathrm{d})$ at $0.15: 1$. The studied fractured surface is parallel to the tensile axis. 


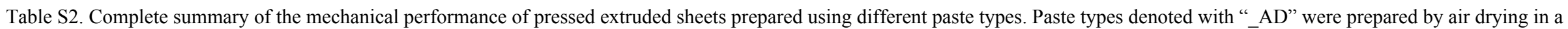

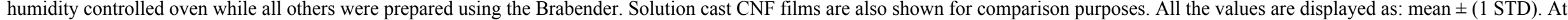
least 6 sample were tested for each case.

\begin{tabular}{|c|c|c|c|c|c|c|c|c|}
\hline Paste Type & $\begin{array}{l}\text { Total Solids } \\
\text { Concentration }\end{array}$ & $\begin{array}{l}\text { Processing aid } \\
\text { to CNF ratio }\end{array}$ & $\begin{array}{l}\text { Ultimate } \\
\text { Strength }\end{array}$ & $\begin{array}{l}\text { Young's } \\
\text { Modulus }\end{array}$ & $\begin{array}{l}\text { Strain to } \\
\text { Failure }\end{array}$ & Density & $\begin{array}{l}\text { Specific } \\
\text { Strength }\end{array}$ & $\begin{array}{l}\text { Specific } \\
\text { Modulus }\end{array}$ \\
\hline & $(w t \%)$ & [aid to $\mathrm{CNF}]^{*}$ & {$[\mathrm{MPa}]$} & {$[\mathrm{GPa}]$} & {$[\%]$} & {$\left[\mathrm{g} / \mathrm{cm}^{\wedge} 3\right]$} & $\mathrm{MPa} /\left(\mathrm{g} / \mathrm{cm}^{\wedge} 3\right)$ & $\mathrm{GPa} /\left(\mathrm{g} / \mathrm{cm}^{\wedge} 3\right)$ \\
\hline Cast CNF & 3 & $0: 1$ & $110.29 \pm 8.23$ & $11.35 \pm 1.5$ & $2.57 \pm 0.60$ & $1.13 \pm 0.11$ & $96.07 \pm 13.38$ & $9.95 \pm 1.77$ \\
\hline $\mathrm{CNF} / \mathrm{CMC}$ & 15.32 & $0.1: 1$ & $110.57 \pm 7.30$ & $9.36 \pm 0.57$ & $1.80 \pm 0.08$ & $1.19 \pm 0.03$ & $93.21 \pm 5.46$ & $7.90 \pm 0.56$ \\
\hline $\mathrm{CNF} / \mathrm{CMC}$ & 19 & $0.1: 1$ & $109.62 \pm 13.10$ & $10.67 \pm 0.87$ & $1.54 \pm 0.30$ & $1.26 \pm 0.02$ & $87.21 \pm 10.15$ & $8.50 \pm 0.73$ \\
\hline $\mathrm{CNF} / \mathrm{CMC}$ & 25 & $0.1: 1$ & $100.84 \pm 14.12$ & $8.57 \pm 0.65$ & $1.29 \pm 0.26$ & $1.37 \pm 0.02$ & $73.87 \pm 10.59$ & $6.27 \pm 0.44$ \\
\hline $\mathrm{CNF} / \mathrm{CMC}$ & 15.32 & $0.15: 1$ & $82.27 \pm 13.30$ & $9.23 \pm 0.91$ & $1.03 \pm 0.22$ & $1.03 \pm 0.13$ & $78.54 \pm 15.11$ & $8.78 \pm 1.12$ \\
\hline CNF/CMC_AD & 15.32 & $0.1: 1$ & $111.23 \pm 9.73$ & $9.48 \pm 0.39$ & $1.41 \pm 0.20$ & $1.32 \pm 0.03$ & $84.55 \pm 6.86$ & $7.21 \pm 0.37$ \\
\hline CNF/CMC_AD & 20 & $0.1: 1$ & $106.93 \pm 16.13$ & $11.03 \pm 0.66$ & $1.16 \pm 0.24$ & $1.36 \pm 0.04$ & $78.91 \pm 11.31$ & $8.15 \pm 0.44$ \\
\hline CNF/CMC_AD & 24 & $0.1: 1$ & $70.35 \pm 7.76$ & $8.24 \pm 0.51$ & $0.80 \pm 0.12$ & $1.36 \pm 0.01$ & $51.56 \pm 5.54$ & $6.04 \pm 0.36$ \\
\hline $\mathrm{CNF} / \mathrm{XG}$ & 15.32 & $0.1: 1$ & $60.08 \pm 19.51$ & $10.01 \pm 0.45$ & $0.75 \pm 0.26$ & $1.24 \pm 0.02$ & $48.58 \pm 16.01$ & $8.08 \pm 0.37$ \\
\hline $\mathrm{CNF} / \mathrm{XG}$ & 15.32 & $0.15: 1$ & $84.53 \pm 10.66$ & $11.32 \pm 0.64$ & $0.79 \pm 0.17$ & $1.28 \pm 0.03$ & $66.20 \pm 8.65$ & $8.86 \pm 0.40$ \\
\hline
\end{tabular}

* The ratio is in terms of dry weight of both components 


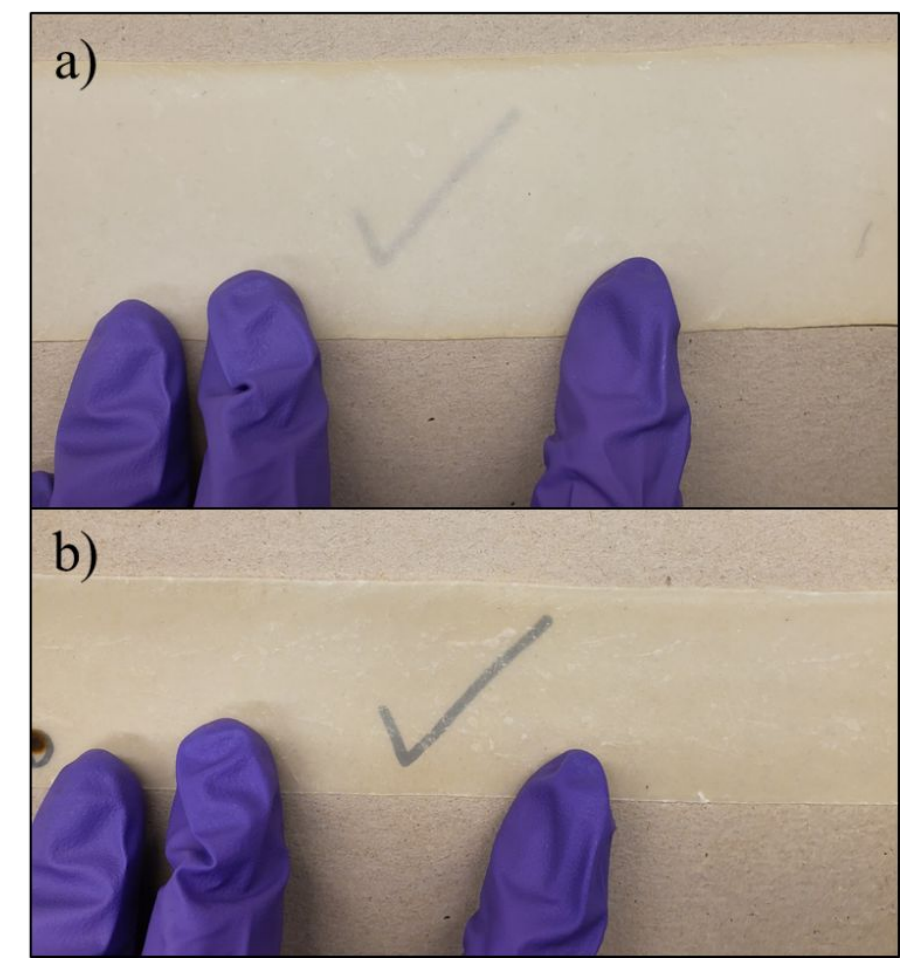

Figure S19. Two different consolidation procedures used to fully dry CNF/CMC extrudates through pressing and heating (a) and heated calendering (b). Note that the check mark can be viewed more easily with the calendered sheet.
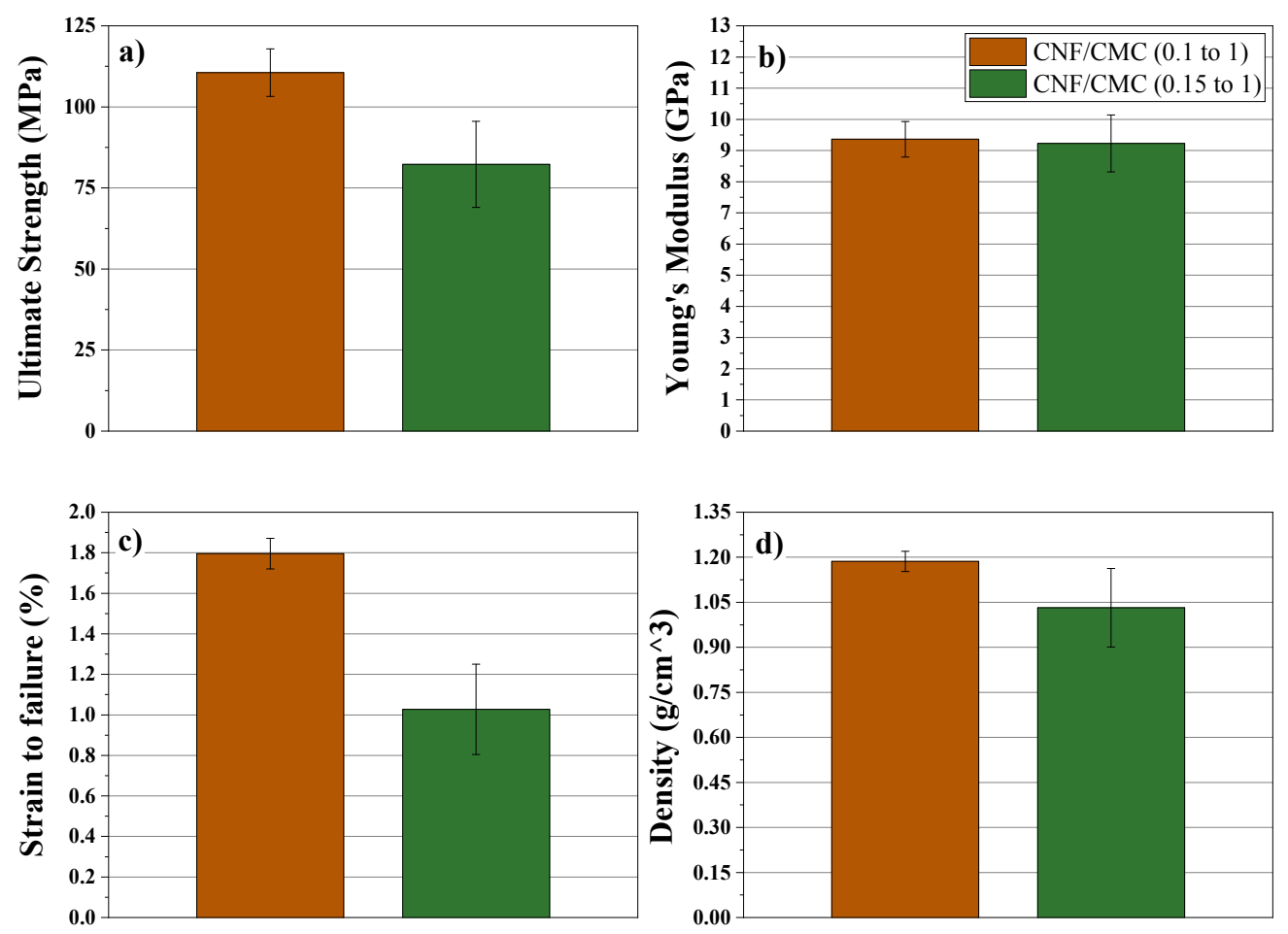

Figure S20. Tensile testing response: ultimate strength (a), Young's modulus (b), strain to failure (c), and density (d) of CNF/CMC pastes at two different dry weight ratios (0.1:1 and 0.15:1). The error bars represent one standard deviation away from the mean. Six samples were tested for each case. 

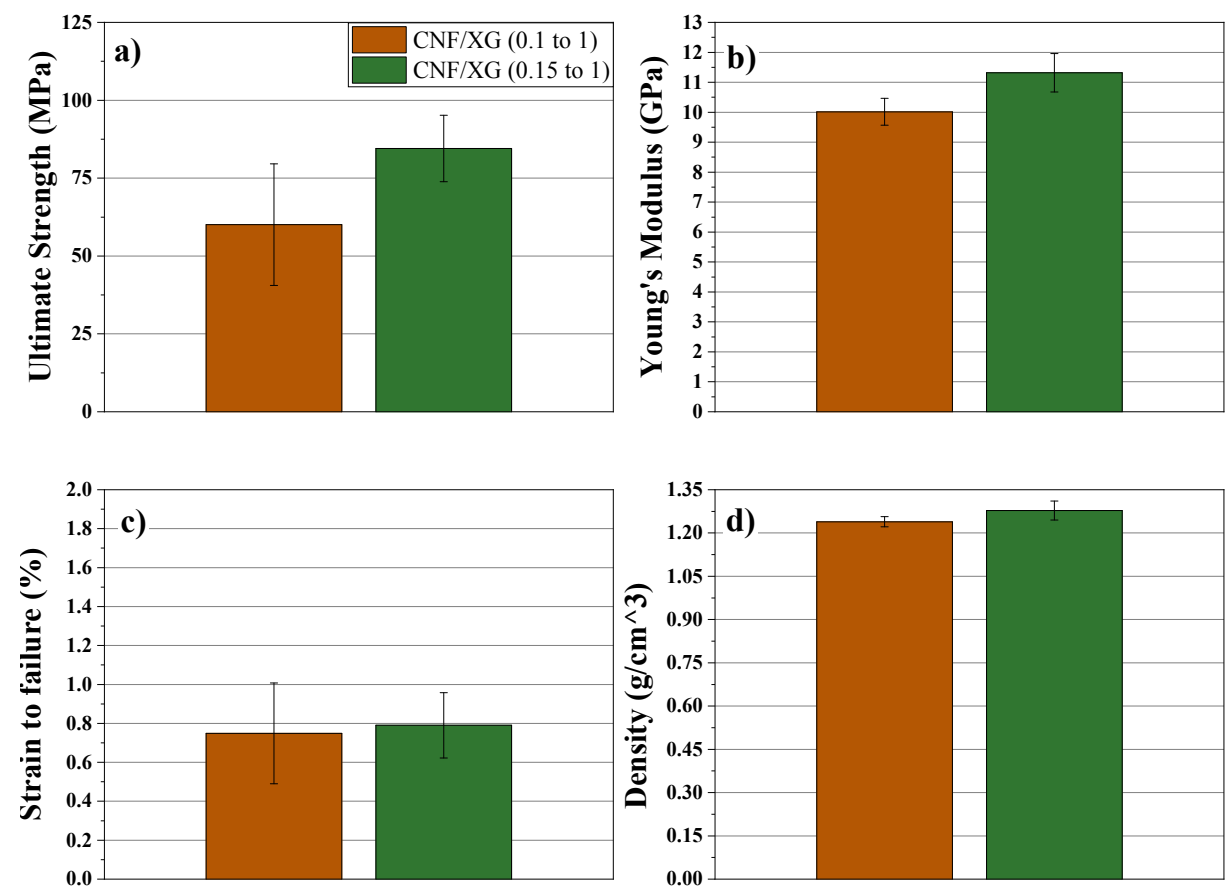

Figure S21. Tensile testing response: ultimate strength (a), Young's modulus (b), strain to failure (c), and density (d) of CNF/XG pastes at two different dry weight ratios $(0.1: 1$ and $0.15: 1)$. The error bars represent one standard deviation away from the mean. Six samples were tested for each case.
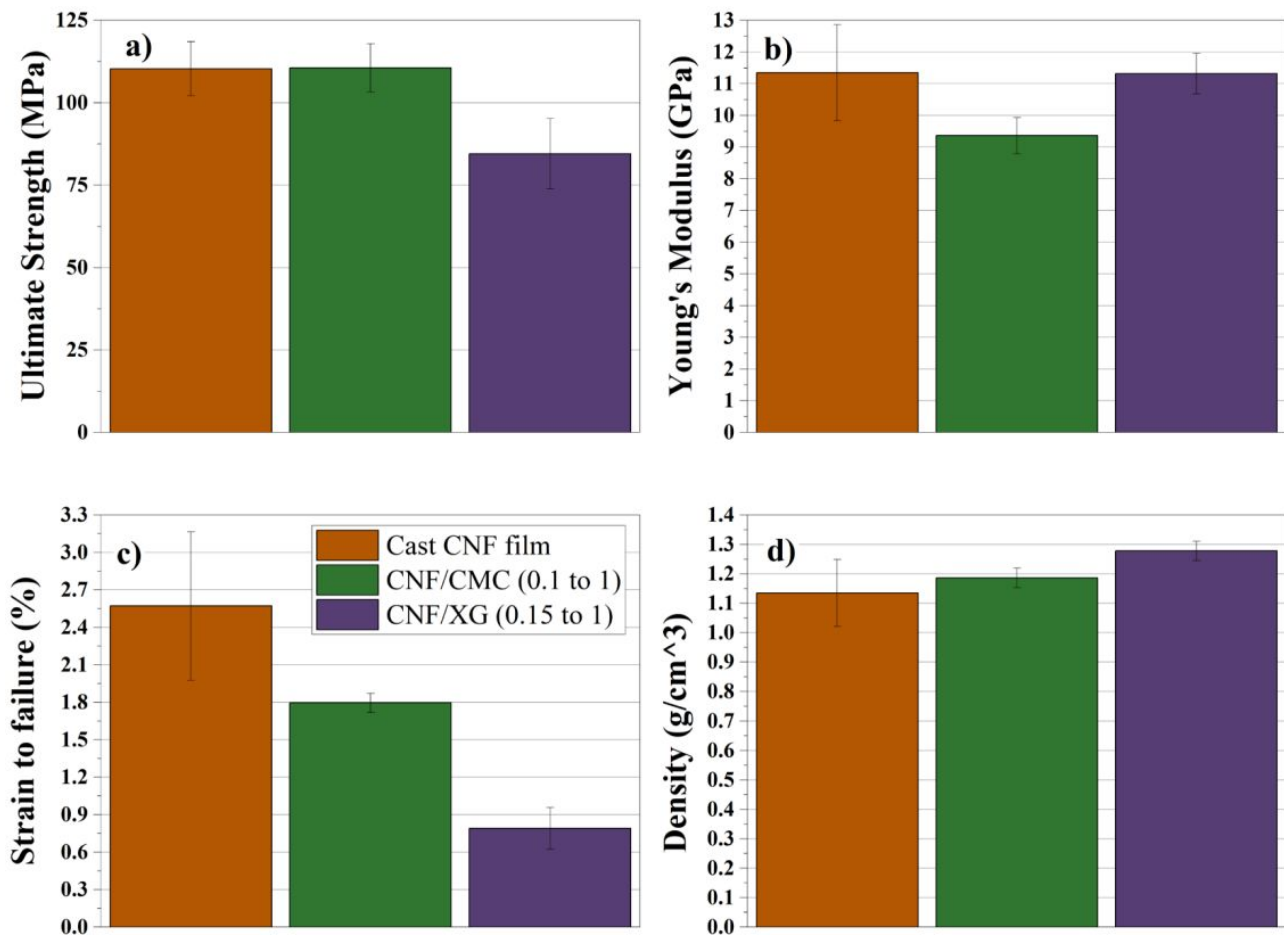

Figure S22. Tensile testing response: ultimate strength (a), Young's modulus (b), strain to failure (c), and density (d) of cast CNF films, extruded CNF/CMC sheets, and extruded CNF/XG sheets at two different processing to CNF dry weight ratios. The error bars represent one standard deviation away from the mean. Seven samples were tested for cast CNF and 6 samples for the extruded sheets. 


\section{Preparation of CNF/CMC air dried pastes}

$\mathrm{CNF} / \mathrm{CMC}$ air dried pastes denoted by "AD" were prepared by first fully dissolving CMC powder in water to reach a concentration of $\sim 2.0 \%(\mathrm{w} / \mathrm{v})$. The viscous CMC solution was then added to a CNF slurry with a solids concentration of $3.1 \mathrm{wt} . \%$ to match a dry weight ratio of $0.1: 1$ (CMC:CNF). Subsequently, the solution was mixed using a planetary SpeedMixerTM (Flacktek Inc.) system at $2500 \mathrm{rpm}$ for 2 minutes and cast into large glass baking trays, crystallization dishes, or $400 \mathrm{ml}$ beakers (Figure S5). The pastes were left to dry slowly inside a humidity-controlled chamber (Memmert $\mathrm{GmbH}+\mathrm{Co} . \mathrm{KG}$, humidity Chamber $\mathrm{HCP} 240$ ) at a temperature of $35^{\circ} \mathrm{C}$, and humidity of $95 \% \mathrm{RH}$ until reaching the desired solids concentration. The shear-mixed CNF/CMC paste preparation is explained in detail in the experimental section of the manuscript.

\section{Validation of Brabender mixing procedure for preparation of CNF/Processing aid pastes}

To verify that the Brabender mixing method is not damaging the CNF fibrils and to show that it is an industrially viable high volume method for preparing pastes for extrusion, CNF/CMC pastes at three solids loadings $(\sim 15, \sim 19$, and $\sim 25 \mathrm{wt} . \%)$ were prepared, extruded, pressed and heated, and tested mechanically in tension. The results from tensile testing are shown in Figure S23. These "mixer-pastes" were compared against air-dried pastes "AD-pastes" with roughly the same solids concentration. The results show that the ultimate strength for $\sim 15 \mathrm{wt} . \%$ and $\sim 19 \mathrm{wt} . \%$ mixer-pastes is equivalent to $\mathrm{AD}$-pastes, while at $\sim 25 \mathrm{wt} . \%$ mixer-pastes outperformed AD-pastes (ultimate strength of $101 \pm 14.12 \mathrm{MPa}$ for mixer-pastes at $\sim 25 \mathrm{wt} \%$ verses $70 \pm 7.76 \mathrm{MPa}$ for AD-pastes at 24 wt.\%), see Table S2. In terms of strain to failure, mixer-pastes outperformed AD-pastes at all concentrations. It is plausible that the decrease in properties for AD-pastes is due to the uneven surface drying that incorporates microscopic aggregates into the sheets once extruded. Yet for both types of processes, the strain to failure decreases with an increase in solids concentration hence lower solids concentration might be optimal for extrusion of tougher sheets. Collectively for both types of pastes (mixer and AD), the density appears to increase as concentration increases and then plateaus at a value of $1.37 \pm 7.76 \mathrm{~g} / \mathrm{cc}$. Furthermore, the Young's modulus of the mixer-pastes and AD-pastes appear to be equivalent to each other at all probed concentrations. Surprisingly for both types of pastes, the modulus appears to vary between $\sim 9 \mathrm{GPa}$ to $\sim 11 \mathrm{GPa}$ as a function of concentration. The reason for the modulus fluctuations are unknown and will be investigated in future publications. 

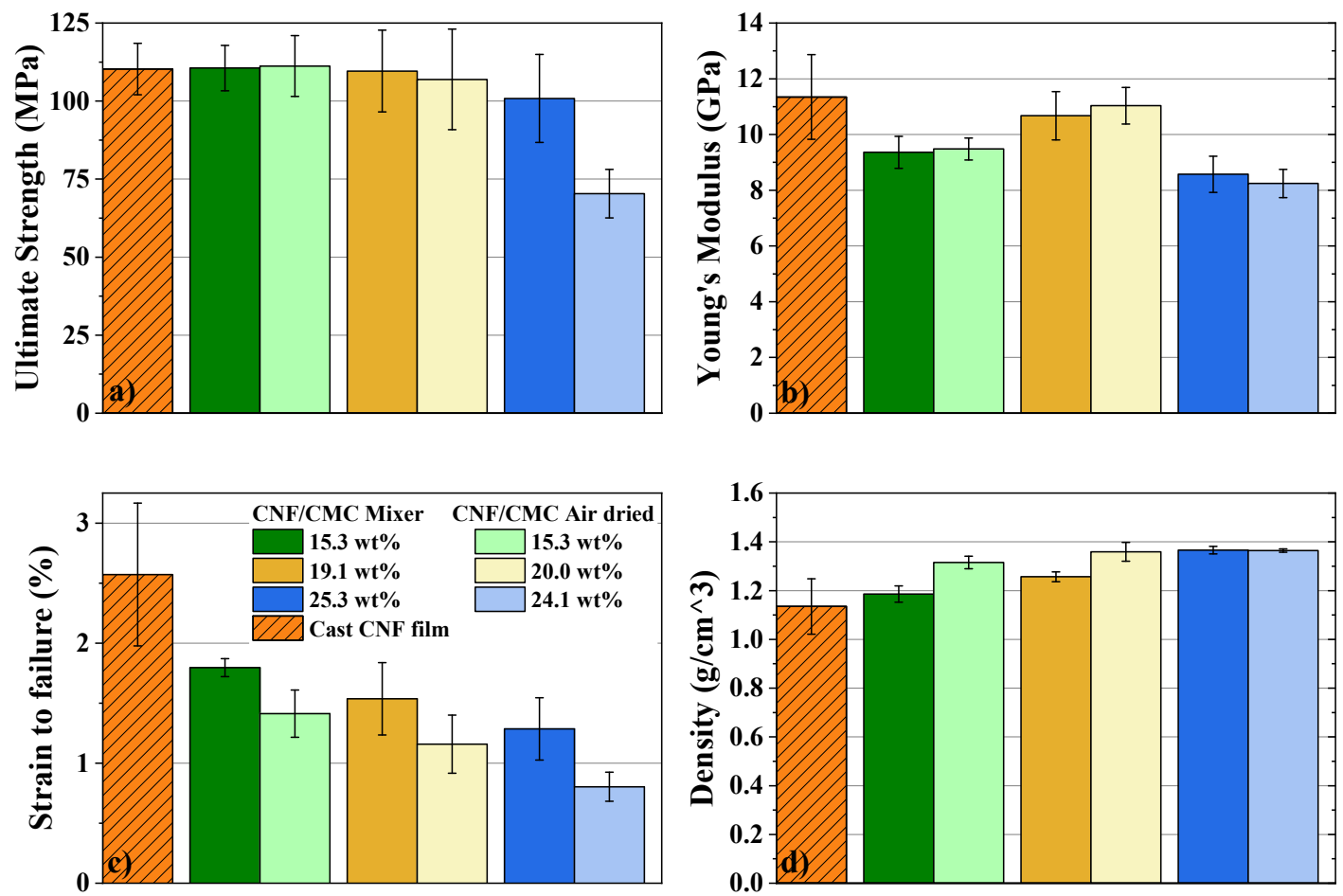

Figure S23. Tensile testing response: ultimate strength (a), Young's modulus (b), strain to failure (c), and density (d) for shearmixed pastes (darker color) verses air-dried pastes (lighter color) prepared using a humidity-controlled oven. The error bars represent one standard deviation away from the mean. The sample size for the cast CNF films was 7 while the sample size for all other cases was 6.

\section{References}

(1) de Britto, D.; Assis, O. B. G. Thermal Degradation of Carboxymethylcellulose in Different Salty Forms. Thermochim. Acta 2009, 494 (1-2), 115-122. https://doi.org/10.1016/j.tca.2009.04.028.

(2) Borsoi, C.; Zimmernnam, M. V. G.; Zattera, A. J.; Santana, R. M. C.; Ferreira, C. A. Thermal Degradation Behavior of Cellulose Nanofibers and Nanowhiskers. J. Therm. Anal. Calorim. 2016, 126 (3), 1867-1878. https://doi.org/10.1007/s10973-016-5653-x.

(3) Brancato, A.; Walsh, F. L.; Sabo, R.; Banerjee, S. Effect of Recycling on the Properties of Paper Surfaces. Ind. Eng. Chem. Res. 2007, 46 (26), 9103-9106. https://doi.org/10.1021/ie070826a.

(4) Saito, T.; Kimura, S.; Nishiyama, Y.; Isogai, A. Cellulose Nanofibers Prepared by TEMPOMediated Oxidation of Native Cellulose. Biomacromolecules 2007, 8 (8), 2485-2491. https://doi.org/10.1021/bm0703970.

(5) Fukuzumi, H.; Saito, T.; Isogai, A. Influence of TEMPO-Oxidized Cellulose Nanofibril Length on Film Properties. Carbohydr. Polym. 2013, 93 (1), 172-177. https://doi.org/10.1016/j.carbpol.2012.04.069. 